Real Analysis Exchange

Vol. 26(1), 2000/2001, pp. 453-456

Inder K. Rana, Department of Mathematics, Indian Institute of Technology, Powai, Mumbai-400076, India. e-mail: ikr@math.iitb.ernet.in

\title{
EXISTENCE OF MEASURES WITH DOMINATED TRANSLATES
}

\begin{abstract}
Let $G$ be a topological group of second category, $\mathcal{B}_{G}$ be its Borel $\sigma$ algebra and $\mathcal{B}$ a $\sigma$-algebra of subsets of $G$ such that $(G, \mathcal{B})$ is a measurable group. For a probability measure $P$ on $(G, \mathcal{B})$, let $P_{g}(E):=P(g E)$ for $g \in G, E \in \mathcal{B}$. The aim of this note is to show that there exists an inner-regular probability measure $P$ and a $\sigma$-finite measure $\mu$ on $(G, \mathcal{B})$ such that $P_{g} \ll \mu \forall g \in G$, iff $G$ is locally-compact and in that case $P_{g} \ll \lambda_{G} \ll \mu \forall g \in G$ on the $\sigma$-algebra $\mathcal{B} \cap \mathcal{B}_{G}$, where $\lambda_{G}$ denotes a Haar measure of $G$.
\end{abstract}

A measurable space $(X, \mathcal{B})$ is said to be standard if there exists a complete separable metric space $Y$ and a bijective map $T: X \rightarrow Y$ such that both $T$ and $T^{-1}$ are measurable with natural Borel $\sigma$-algebra on $Y$. By a measurable group $(G, \mathcal{B})$ we mean that $G$ is a group and $\mathcal{B}$ is a $\sigma$-algebra of subsets of $G$ such that the map $(g, h) \mapsto g h^{-1}$ from $G \times G \rightarrow G$ is measurable when $G \times G$ is given the product $\sigma$-algebra $\mathcal{B} \times \mathcal{B}$. Let $(G, \mathcal{B})$ be a measurable group. A measure $\mu$ on $(G, \mathcal{B})$ is called (left) quasi-invariant if $\forall E \in \mathcal{B}, \mu(E)=0 \Leftrightarrow \mu_{g}(E):=$ $\mu(g E)=0 \forall g \in G$. We say $\mu$ has dominated translates if there exists a $\sigma$-finite measure $\nu$ on $(G, \mathcal{B})$ such that $\mu_{g} \ll \nu \forall g \in G$. The well-known theorem due to G.W. Mackey (refer [2]) says that there exist a quasi-invariant measure on a standard measurable group $(G, \mathcal{B})$ iff $G$ is a locally-compact group under some topology $\tau$, and in that case the Borel $\sigma$-algebra given by $\tau$ is $\mathcal{B}$ with $\mu \equiv \lambda_{G}$, the (left) Haar measure on $G$. In [4], the following generalization of this result is proved :

Theorem 1. Let $G$ be a topological group of second category and having cardinality almost that of the continuum. If for some $\sigma$-algebra $\mathcal{B}$ of subsets of $G$, such that $(G, \mathcal{B})$ is a measurable group, there exists an inner-regular probability measure $P$ on $(G, \mathcal{B})$ having dominated translates, then $G$ is locally-compact.

Key Words: Measurable group, inner-regular measures, quasi-invariant measures.

Mathematical Reviews subject classification: 43A05

Received by the editors March 3, 2000 
Further, if $\mathcal{B}_{G}$ is the $\sigma$-algebra of Borel subsets of $G$ and $\lambda_{G}$ is a Haar measure of $G$, then $P_{g} \ll \lambda_{G} \forall g \in G$ on the $\sigma$-algebra $\mathcal{B} \cap \mathcal{B}_{G}$.

The proof of the above theorem assumed the continuum hypothesis. In the present note we give a simple argument that avoids the use of the continuum hypothesis and extends the above theorem to all groups of second category. The statement of the following lemma can be found in [1].

For this discussion, the key result is due to Halmos-Savage and is given in the next lemma. We include a proof which uses an argument similar to those used in [1].

Lemma 1. Let $(X, \mathcal{B})$ be a measurable space and let $\mathcal{P}=\left\{P_{\theta}\right\}_{\theta \in \Theta}$ be any family of probability measures on $(X, \mathcal{B})$. If there exists a $\sigma$-finite measure $\mu$ on $(X, \mathcal{B})$ such that $P_{\theta} \ll \mu \forall \theta \in \Theta$, then there exists a countable sub-family $\mathcal{Q}=\left\{P_{\theta_{n}}\right\}_{n \geq 1}$ of $\mathcal{P}$ such that for $E \in \mathcal{B}, P_{\theta}(E)=0 \Leftrightarrow P_{\theta_{n}}(E)=0 \quad \forall n \geq 1$ (we write this as $\mathcal{P} \equiv \mathcal{Q}$ ).

Proof. Without loss of generality we may assume that $\mu$ is finite. For every $\theta \in \Theta$, let $S_{\theta}=\left\{x \in X \mid \frac{d P_{\theta}}{d \mu}(x)>0\right\}$. Let $C \in \mathcal{B}$ be called a chain if $C$ is a countable union of sets from $\mathcal{B}$, each of which is a subset of some $S_{\theta}$. Let $\alpha=\sup \{\mu(C) \mid C$ a chain $\}$. Clearly $\alpha$ is finite and is in fact attained since countable union of chains is a chain. Let $\alpha=\mu(C)$, where $C=\bigcup_{n=1}^{\infty} B_{n}$ and $B_{n} \subseteq S_{\theta_{n}}$. Let $\mathcal{Q}=\left\{P_{\theta_{n}} \mid n=1,2, \ldots\right\}$. We show that $\mathcal{Q}$ is the required subfamily. Let $P_{\theta_{n}}(A)=0 \forall n \geq 1$. Then $\mu\left(A \cap S_{\theta_{n}}\right)=0$ and hence

$$
\mu(A \cap C)=0 \text { as } C \subseteq \bigcup_{n=1}^{\infty} B_{n} \subseteq \bigcup_{n=1}^{\infty} S_{n} .
$$

This implies that $P_{\theta}(A \cap C)=0$ for all $\theta \in \Theta$. Also if $P_{\theta}(A-C)>0$ for some $\theta$, then $P_{\theta}\left((A-C) \cap S_{\theta}\right)>0$. Hence $C^{\prime}=C \cup\left((A-C) \cap S_{\theta}\right)$ is a chain with $\mu\left(C^{\prime}\right)>\mu(C)$, contradicting the maximality of $\mu(C)$. Hence $P_{\theta}(A-C)=0 \forall \theta \in \Theta$. This proves that $\mathcal{Q} \equiv \mathcal{P}$.

The following lemma can be found in [3]

Lemma 2. Let $G$ be a topological group of second category. Then there exists a locally-finite inner-regular quasi-invariant measure on a $\sigma$-field $\mathcal{B}$ of subsets of $G$ such that $(G, \mathcal{B})$ is a measurable group, if and only if $G$ is locally-compact.

Using the results listed above, we prove the following :

Theorem 2. Let $G$ be a topological group of second category and $\mathcal{B}$ be a $\sigma$-field $\mathcal{B}$ of subsets of $G$ such that $(G, \mathcal{B})$ is a measurable group. Then there exists 
an inner-regular probability measure $P$ on $(G, \mathcal{B})$ with dominated translates if and only if $G$ is locally-compact. In that case $\forall g \in G, P_{g} \ll \lambda_{G}$ on the $\sigma$-algebra $\mathcal{B}_{G} \cap \mathcal{B}$, where $\mathcal{B}_{G}$ is the $\sigma$-algebra of Borel subsets of $G$ and $\lambda_{G}$ is a Haar-measure on $G$.

Proof. Obviously, if $G$ is locally-compact and $\lambda_{G}$ denotes a Haar measure, then with $\mathcal{B}=B_{G}$, the $\sigma$-algebra of Borel subsets of $G$, and $P=\mu$, some probability measure equivalent to $\lambda_{G}$, the required properties hold.

Conversely, let $\mathcal{B}$ be a $\sigma$-algebra of subsets of $G$ such that $(G, \mathcal{B})$ is a measurable group. Further, let there exist a probability $P$ having dominated translates, i.e., there exists a $\sigma$-finite measure $\mu$ on $(G, \mathcal{B})$ such that $P_{g} \ll$ $\mu \forall g \in G$. We first show that there exists a quasi-invariant probability measure on $(G, \mathcal{B})$. By Lemma 1 , there exists a countable sub-family $\mathcal{Q}=$ $\left\{P_{g_{i}} \mid i=1,2, \ldots\right\}$ of $\mathcal{P}$ such that $\mathcal{P} \equiv \mathcal{Q}$. Define $\nu:=\sum_{i=1}^{\infty} \frac{P_{g_{i}}}{2^{i}}$. It is to check that $\nu$ is an inner-regular probability measure on $(G, \mathcal{B})$. Further $\nu$ is quasiinvariant. Now by Lemma $2, G$ is locally-compact. Let $\mathcal{B}_{G}$ denote the Borel $\sigma$-algebra of $G$ and $\lambda_{G}$ denote a Haar measure on $G$. Then $\lambda_{G}$ and $\nu$ are two quasi-invariant measures on $\left(\mathcal{B}_{G} \cap \mathcal{B}\right)$. Hence $\lambda_{G} \equiv \nu$. Clearly $\mu \gg \nu$, and hence $\mu \gg \lambda_{G} \gg P_{g} \forall g \in G$ on $\mathcal{S} \cap \mathcal{B}$.

\section{References}

[1] Patrick Billingsley, Probability and measure, (2nd Edition), John Wiley and Sons, New York 1986.

[2] Q. J. Burrel, Standard spaces with a quasi-invariant measure, J. Lond. Math. Soc. 2(1970), 627-634.

[3] Kai Wang Ng, Quasi-invariant measures on groups of second category, J. London Math. Soc. 7(1973), 170-174.

[4] I. K. Rana, On a characterization of locally compact groups of second category, assuming the continuum hypothesis, Proc. Amer. Math. Soc. 64(1977), 97-100. 
